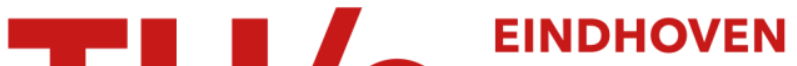 UNIVERSITY OF TECHNOLOGY
}

\section{Efficient state reference generation for torque control in externally excited synchronous machines}

\section{Citation for published version (APA):}

Carpiuc, S. C., \& Lazar, M. (2015). Efficient state reference generation for torque control in externally excited synchronous machines. Journal of Dynamic Systems, Measurement and Control : Transactions of the ASME, 137(5), 051015-1/7. [051015]. https://doi.org/10.1115/1.4029243

DOI:

$10.1115 / 1.4029243$

Document status and date:

Published: 01/01/2015

\section{Document Version:}

Publisher's PDF, also known as Version of Record (includes final page, issue and volume numbers)

\section{Please check the document version of this publication:}

- A submitted manuscript is the version of the article upon submission and before peer-review. There can be important differences between the submitted version and the official published version of record. People interested in the research are advised to contact the author for the final version of the publication, or visit the $\mathrm{DOI}$ to the publisher's website.

- The final author version and the galley proof are versions of the publication after peer review.

- The final published version features the final layout of the paper including the volume, issue and page numbers.

Link to publication

\section{General rights}

Copyright and moral rights for the publications made accessible in the public portal are retained by the authors and/or other copyright owners and it is a condition of accessing publications that users recognise and abide by the legal requirements associated with these rights.

- Users may download and print one copy of any publication from the public portal for the purpose of private study or research.

- You may not further distribute the material or use it for any profit-making activity or commercial gain

- You may freely distribute the URL identifying the publication in the public portal.

If the publication is distributed under the terms of Article 25fa of the Dutch Copyright Act, indicated by the "Taverne" license above, please follow below link for the End User Agreement:

www.tue.nl/taverne

Take down policy

If you believe that this document breaches copyright please contact us at:

openaccess@tue.nl

providing details and we will investigate your claim. 


\section{Sabin-Constantin Carpiuc \\ Department of Automatic Control \\ and Applied Informatics, \\ "Gheorghe Asachi" Technical University of lasi, lasi 700050, Romania \\ e-mail: scarpiuc@ac.tuiasi.ro \\ Mircea Lazar \\ Department of Electrical Engineering, Eindhoven University of Technology, Eindhoven 5600MB, The Netherlands e-mail: m.lazar@tue.nl}

\section{Efficient State Reference Generation for Torque Control in Externally Excited Synchronous Machines}

A relevant challenge in hybrid electric vehicles (HEVs) and full EVs is the torque control of externally excited synchronous machines (EESMs). Effective torque control requires an efficient solution to the state reference generation problem, which is a nonlinear nonconvex optimization problem. The goal of this paper is to develop a state reference generation algorithm based on the gridding of the state and output spaces. First, an approximation defined over a cubic partition of the torque function with a piecewise affine (PWA) function is made. As a result, the state reference generation problem is reduced in each cube to solving a convex optimization problem. Moreover, this approach provides guarantees about the error bound introduced by the state reference generation procedure for the full operational state-space. To illustrate the effectiveness and robustness of the proposed algorithm, several real-time results obtained on an industrial hardware-in-the-loop $(H I L)$ test-bench are presented. The obtained results show significant improvement compared with existing state-of-the-art reference generation methods. [DOI: $10.1115 / 1.4029243]$

\section{Introduction}

In the last decades, electric drives have seen major changes due to the development of power electronics. These changes made it possible to successfully use electric drives in most automotive applications. The focus on high efficiency drives has led to the adoption of synchronous machines. The EESM is a good choice for the automotive industry due to its many advantages: high efficiency, high power density, high torque at start-up [1], high reliability, and an additional degree-of-freedom compared with the permanent magnet synchronous machine (PMSM) [2,3]. Therefore, the EESM is more appropriate for applications such as HEVs and full EVs as a traction machine.

For a better understanding and for control synthesis, the EESM can be analyzed using the rotor reference frame $(d-q$ reference frame). The stator quantities (voltages, currents, and flux linkages) can be transformed in the rotor reference frame and vice versa by using Park transformations [4-6]. In the resulting model, the torque can be controlled in a similar way as for a DC machine. The main challenge for traction electric drives is to control the torque of the electric machine and in the same time to ensure high overall efficiency in a wide speed range. In field oriented control (FOC) [7] schemes for EESMs, it is important to control the state vector, represented by the stator and excitation currents in the rotor reference frame. In this case, the torque control problem is reduced to a state vector control problem. As such, the control strategy has to compute also the state reference, which involves solving a nonlinear nonconvex optimization problem. Therefore, state reference generation is a crucial problem for automotive electrical traction drives where the electric machine has to be able to work efficiently in a wide speed-torque range, see, for example, Refs. $[1,3,8,9]$. This motivates the need for real-time implementable state reference generation algorithms for automotive electrical traction drives.

Contributed by the Dynamic Systems Division of ASME for publication in the Journal of Dynamic Systems, Measurement, and Control. Manuscript received August 29, 2013; final manuscript received November 19, 2014; published online January 27, 2015. Assoc. Editor: Luis Alvarez.
During the last few years, this topic has been actively studied but with focus on the PMSM torque control, which can be considered a particular case of the EESM torque control. For an overview, the interested reader is referred to Refs. [10-12], and the references therein. As such, a possible approach is to adapt the existing state reference generation strategies developed for PMSM torque control. However, this approach is applicable to the EESM only under some assumptions and it is difficult to take into account the variation of the machine parameters. Another approach, which is directly applicable to the EESM, is based on solving offline the nonlinear nonconvex state reference generation problem, while minimizing the machine power losses $[3,8]$. This allows an increase in efficiency when compared to the torque maximization strategy proposed in Ref. [9].

However, all of the above state reference generation strategies provide only a heuristic solution, which is not easily applicable for different parameters and do not provide any guarantees regarding bounds on the error introduced by the state reference generation. Therefore, the goal of this paper is to provide a systematic solution for the EESM state reference generation, which is generally applicable and it guarantees a bounded reference error. To achieve this goal, the output function is approximated locally with a PWA function over a cubic partition of the state-space. The choice of a cubic partition is justified by the fact that it allows fast point location, which is crucial in applications with tight time constraints such as EESM drives. Then, it is shown that the state reference vector can be obtained by solving a linear program (LP). It is then proven that using this approach an error bound is guaranteed for the full operational state-space. Moreover, the error bound can be reduced by applying the method of Refs. $[3,8,9]$ in a vicinity of the obtained solution, which significantly reduces the exploration space. Also, this algorithm can be easily applied to other machines and parameters and can ensure minimal power losses. The proposed method in conjunction with a discrete-time linear quadratic regulator (LQR) is tested on an industrial HIL test-bench using a real-time implementation in a microcontrollerbased electronic control unit (ECU). HIL testing is a common choice for testing and validation of automotive control systems in real-time [13,14]. Moreover, in testing the developed algorithms, 
realistic real-time requirements and limitations for the automotive industry [15] were utilized.

A preliminary version of the results presented in this paper was reported in the conference paper [16]. Therein, the proposed state reference generation algorithm was tested only in SIMULINK, for the same plant model as the model used for reference calculation. Moreover, therein, an optimal continuous-time controller was employed for driving the system states to the reference. This is not compatible with real-implementation in automotive ECUs, which involve a digital control architecture. In contrast, this article presents a digital control architecture that can be implemented in automotive ECUs. The developed control architecture was implemented and successfully tested on an industrial HIL testbench and it was certified for industrial usage according to the automotive industry real-time standards.

The remainder of the paper is organized as follows: The EESM model and problem formulation are presented in Sec. 2. The algorithm for the state reference generation is presented in Sec. 3. The digital control architecture and real-time experimental results are presented and analyzed in Sec. 4 and conclusions are drawn in Sec. 5 .

1.1 Basic Notations and Definitions. Let $\mathbb{R}, \mathbb{R}_{+}, \mathbb{Z}$, and $\mathbb{Z}_{+}$ denote the field of real numbers, the set of non-negative reals, the set of integer numbers, and the set of non-negative integers, respectively. For every $c \in \mathbb{R}$ and $\Pi \subseteq \mathbb{R}$ define $\Pi_{\geq c}:=\{k \in \Pi \mid$ $k \geq c\}$ and similarly $\Pi_{\leq c}, \mathbb{R}_{\Pi}:=\Pi$ and $\mathbb{Z}_{\Pi}:=\overline{\mathbb{Z}} \cap \Pi$. For a vector $x \in \mathbb{R}^{n},[x]_{i}$ denotes the $i$ th element of $x$ and $|\cdot|$ denotes the absolute value. For a matrix $Z \in \mathbb{R}^{n \times m},[Z]_{i j} \in \mathbb{R}$ denotes the element on the $i$ th row and $j$ th column of $Z$. $I_{n} \in \mathbb{R}^{n \times n}$ denotes the $n$th dimensional identity matrix. $O_{n} \in \mathbb{R}^{n \times n}$ denotes the $n$th dimensional zero matrix. $t \in \mathbb{R}_{+}$denotes the continuous-time variable.

\section{EESM Model and Problem Formulation}

2.1 EESM Model. The $d-q$ equivalent circuit of a threephase EESM is presented in Fig. 1.

From Fig. 1, the EESM voltage equations are obtained by applying Kirchhoff's voltage law

$$
\begin{gathered}
v_{d s}(t)=R_{s} i_{d s}(t)+L_{d} \frac{d i_{d s}(t)}{d t}+M_{d} \frac{d i_{e}(t)}{d t}-\omega_{e}(t) L_{q} i_{q s}(t) \\
v_{q s}(t)=R_{s} i_{q s}(t)+L_{q} \frac{d i_{q s}(t)}{d t}+\omega_{e}(t)\left(L_{d} i_{d s}(t)+M_{d} i_{e}(t)\right) \\
v_{e}(t)=R_{e} i_{e}(t)+L_{e} \frac{d i_{e}(t)}{d t}+M_{d} \frac{d i_{d s}(t)}{d t}
\end{gathered}
$$

where $R_{s}$ and $R_{e}$ are the stator and excitation windings resistance; $v_{d s}$ and $v_{q s}$ are the $d$ and $q$ axis stator voltages; $i_{d s}$ and $i_{q s}$ are the

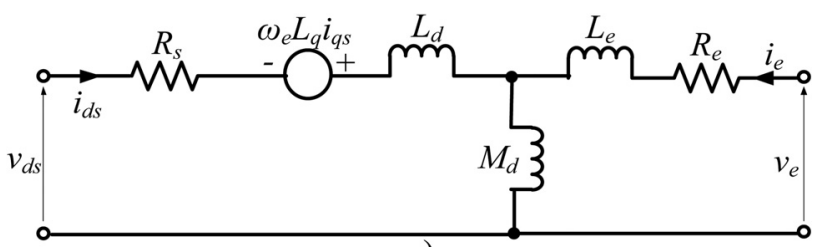

a)

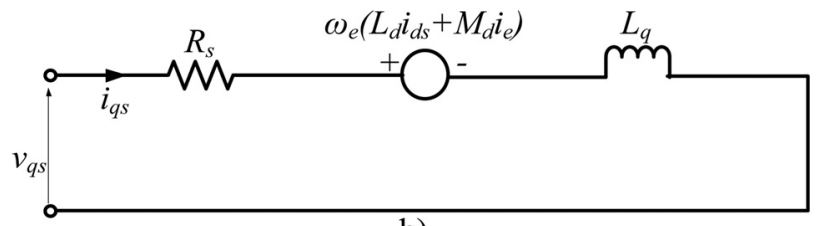

b)

Fig. 1 EESM $d-q$ equivalent circuit: (a) Direct-axis equivalent circuit and (b) Quadrature-axis equivalent circuit. $d$ - and $q$-axis stator currents; $L_{d}$ and $L_{q}$ are the $d$ and $q$ axis inductances; $M_{d}$ is the mutual inductance between stator and rotor windings; $v_{e}$ is the excitation voltage; $i_{e}$ is the excitation current; $L_{e}$ is the excitation inductance; and $\omega_{e}$ is the rotor electrical angular velocity.

From the input power in terms of currents in the rotor reference frame, the electromagnetic torque function results

$$
T_{e}(t)=\frac{3 P_{p}}{2}\left[M_{d} i_{q s}(t) i_{e}(t)+\left(L_{d}-L_{q}\right) i_{d s}(t) i_{q s}(t)\right]
$$

where $P_{p}$ denotes the number of pole pairs.

By introducing the stator currents in the rotor reference frame and the excitation current as state variables, i.e.,

$$
x(t):=\left[\begin{array}{lll}
i_{d s}(t) & i_{q s}(t) & i_{e}(t)
\end{array}\right]^{\mathrm{T}}
$$

choosing as control inputs the stator voltages in the rotor reference frame and the excitation voltage, i.e.,

$$
u(t):=\left[\begin{array}{lll}
v_{d s}(t) & v_{q s}(t) & v_{e}(t)
\end{array}\right]^{\mathrm{T}}
$$

choosing as output the electromagnetic torque, i.e.,

$$
y(t):=T_{e}(t)
$$

and considering the rotor electrical angular velocity, $\omega_{e}(t)$, as a variable parameter, from Eqs. (1) and (2), the EESM state-space model is obtained [17], i.e.,

$$
\begin{gathered}
\dot{x}(t)=A\left(\omega_{e}(t)\right) x(t)+B u(t) \\
y(t)=x(t)^{\mathrm{T}} C x(t)
\end{gathered}
$$

where

$$
\begin{aligned}
& A\left(\omega_{e}(t)\right)=\left[\begin{array}{ccc}
\frac{L_{e} R_{s}}{M_{d}^{2}-L_{d} L_{e}} & -\frac{L_{q} L_{e} \omega_{e}(t)}{M_{d}^{2}-L_{d} L_{e}} & -\frac{M_{d} R_{e}}{M_{d}^{2}-L_{d} L_{e}} \\
-\frac{L_{d} \omega_{e}(t)}{L q} & -\frac{R_{s}}{L_{q}} & -\frac{M_{d} \omega_{e}(t)}{L_{q}} \\
-\frac{M_{d} R_{s}}{M_{d}^{2}-L_{d} L_{e}} & \frac{M_{d} L_{q} \omega_{e}(t)}{M_{d}^{2}-L_{d} L_{e}} & \frac{L_{d} R_{e}}{M_{d}^{2}-L_{d} L_{e}}
\end{array}\right] \\
& B=\left[\begin{array}{ccc}
-\frac{L e}{M_{d}^{2}-L_{d} L_{e}} & 0 & \frac{M_{d}}{M_{d}^{2}-L_{d} L_{e}} \\
0 & \frac{1}{L_{q}} & 0 \\
\frac{M_{d}}{M_{d}^{2}-L_{d} L_{e}} & 0 & -\frac{L_{d}}{M_{d}^{2}-L_{d} L_{e}}
\end{array}\right] \\
& C=\left[\begin{array}{ccc}
0 & \frac{3 P_{p}\left(L_{d}-L_{q}\right)}{4} & 0 \\
\frac{3 P_{p}\left(L_{d}-L_{q}\right)}{4} & 0 & \frac{3 P_{p} M_{d}}{4} \\
0 & \frac{3 P_{p} M_{d}}{4} & 0
\end{array}\right]
\end{aligned}
$$

In what follows, the time symbol $t$ will be omitted in order to reduce the notational complexity. The system matrices depend on the machine parameter, $\beta=\left[\begin{array}{lll}L_{d} & L_{q} & M_{d}\end{array}\right]^{\mathrm{T}}$. The parameter $\beta$ is also omitted to reduce the notational complexity.

2.2 Physical Limits. As most automotive applications, the controlled system is subject to several constraints that originate from physical limits or performance requirements. More specifically, we have 


$$
\begin{gathered}
\sqrt{[x]_{1}^{2}+[x]_{2}^{2}} \leq I_{s, \max } \\
\sqrt{[u]_{1}^{2}+[u]_{2}^{2}} \leq U_{s, \max } \\
{\left[x_{\min }\right]_{i} \leq[x]_{i} \leq\left[x_{\max }\right]_{i}, \quad i \in \mathbb{Z}_{[1,3]}} \\
-T_{\max } \leq y \leq T_{\max }
\end{gathered}
$$

where $I_{s, \max }$ and $U_{s, \max }$ are the available maximum phase stator current and voltage, $x_{\min }$ and $x_{\max }$ are the minimum and the maximum admissible currents, and $T_{\max }$ is the physical torque limit of the machine.

The voltage constraint described by Eq. (4b) can be rewritten in terms of state variables using the steady-state descriptions of Eq. ( $3 a)$ and neglecting the voltage drop across the stator resistance, i.e.,

$$
\left(L_{q}[x]_{2}\right)^{2}+\left(L_{d}[x]_{1}+M_{d}[x]_{3}\right)^{2}-\left(\frac{U_{s, \max }}{\omega_{e}}\right)^{2} \leq 0
$$

where $U_{s, \max }=\left(V_{\mathrm{DC}} / \sqrt{3}\right)$ in the case when the space vector pulse width modulation is employed for controlling the power inverter, where $V_{\mathrm{DC}}$ is the available direct current (DC) link voltage.

In what follows, we consider a cubic subset of the state-space, denoted by $\mathbb{X}$, which is defined by Eq. $(4 c)$ and an interval of the output space denoted by $\mathbb{Y}$, which is defined by Eq. (4d). Due to physical considerations, $\mathbb{X}$ will always contain the zero point and similarly, $\mathbb{Y}$ will also contain zero. Moreover, the constraints Eqs. (4a) and (4b), which results in constraint Eq. (5) in the statespace, define (possibly degenerate) ellipsoids that contain zero. Therefore, the admissible operational state-space region, defined by the intersection of $\mathbb{X}$ with the subsets of the state-space induced by constraints Eqs. $(4 a)$, $(4 d)$, and (5), is always nonempty and contains the zero point.

2.3 Problem Formulation. The goal of the EESM torque control problem is to enable efficient state reference generation and to control the state vector. In this paper, only the state reference generation problem is addressed. Throughout the paper, it is assumed that the output reference $y_{\text {ref }} \in \mathbb{Y}$, represented by the torque reference, is available. As such, the state reference generation problem can be formulated as follows.

Problem 1. Given the output function Eq. (3b), the set $\mathbb{X}$ and the system reference $y_{\mathrm{ref}} \in \mathbb{Y}$ generate the state reference vector by solving the following optimization problem:

$$
\min _{x} 0
$$

subject to Eqs. (4a), (5) and

$$
\begin{gathered}
x^{\top} C x-y_{\text {ref }}=0 \\
x \in \mathbb{X}
\end{gathered}
$$

Because the system output function described by Eq. (3b) has a nonunique solution, the overall machine efficiency can be increased by minimizing the machine power losses $P_{\text {loss }}(x)$. The power losses model is given in Appendix A.

\section{State Reference Generation}

The idea presented in this paper is based on a PWA approximation of the output function described by Eq. $(3 b)$. For this, we use an algorithm based on partitioning the state-space into a finite number of cubes, i.e., $\left\{\mathcal{C}_{i}\right\}_{i \in \mathbb{Z}_{[1, n]}}$ where $n \in \mathbb{Z}_{\geq 1}$ and $\mathcal{C}_{i}$ : $=\left\{x \in \mathbb{X} \mid M_{i} x \leq m_{i}\right\}$ with $M_{i} \in \mathbb{R}^{6 \times 3}, m_{i} \in \mathbb{R}^{6}$. The cubic partition was employed because it allows efficient point location.

To avoid solving the nonlinear nonconvex optimization problem described in Problem 1, for every cube $\mathcal{C}_{i}$ we approximate the output function described by Eq. ( $3 b$ ) with an affine function, i.e.,

$$
f_{i}(x):=H_{i} x+h_{i},
$$

where $x \in \mathcal{C}_{i}, H_{i} \in \mathbb{R}^{1 \times 3}, h_{i} \in \mathbb{R}$, and $i \in \mathbb{Z}_{[1, n]}$.

The affine function described by Eq. (7) is determined by solving offline an optimization problem which can be recast as a LP for each $\mathcal{C}_{i}$, i.e.,

$$
\begin{gathered}
\min _{\left\{\varepsilon_{i}(j, l)\right\}_{(j, l) \in \mathbb{Z}_{[1,8]}^{2}}} \max _{(j, l) \in \mathbb{Z}_{[1,8]}^{2}} \varepsilon_{i}(j, l) \\
\text { subject to } \\
\pm\left(\left(H_{i}-x_{l}^{\top} C\right) x_{j}+h_{i}\right) \leq \varepsilon_{i}(j, l), \quad \forall(j, l) \in \mathbb{Z}_{[1,8]}^{2}
\end{gathered}
$$

Next, let us define $e_{i}:=\max _{(j, l) \in \mathbb{Z}_{[1,8]}^{2}} \varepsilon_{i}(j, l)$. Using the same reasoning as in Theorem IV.2 of Ref. [18], the following result is obtained.

Lemma 1. Let $i \in \mathbb{Z}_{[1, n]}, l \in \mathbb{Z}_{[1,8]}, C \in \mathbb{R}^{3 \times 3}, H_{i} \in \mathbb{R}^{1 \times 3}$, and $h_{i} \in \mathbb{R}$ be given. Let $\left\{x_{j}\right\}_{j \in \mathbb{Z}_{[1,8]}}$ be the vertices of $\mathcal{C}_{i}$. If it holds that

$$
\pm\left(\left(H_{i}-x_{l}^{\top} C\right) x_{j}+h_{i}\right) \leq e_{i}, \quad \forall(j, l) \in \mathbb{Z}_{[1,8]}
$$

then it holds that

$$
\left|\left(H_{i}-x^{\top} C\right) x+h_{i}\right| \leq e_{i}, \quad \forall x \in \mathcal{C}_{i}
$$

Proof. From Eq. (9), it results immediately that

$$
\left|\left(H_{i}-x_{l}^{\mathrm{T}} C\right) x_{j}+h_{i}\right| \leq e_{i}, \quad \forall(j, l) \in \mathbb{Z}_{[1,8]}^{2}
$$

Next, let $j \in \mathbb{Z}_{[1,8]}$ be fixed. Then, $\forall x \in \mathcal{C}_{i}, \exists \alpha_{l} \in \mathbb{R}_{[0,1]}$, $l \in \mathbb{Z}_{[1,8]}$ with $\sum_{l=1}^{8} \alpha_{l} x_{l}=x$ and $\sum_{l=1}^{8} \alpha_{l}=1$. Left multiplying Eq. (11) with $\alpha_{l}>0$, for each $l \in \mathbb{Z}_{[1,8]}$ and summing up the resulting inequalities yields

$$
\left|\left(H_{i}-x^{\mathrm{T}} C\right) x_{j}+h_{i}\right| \leq e_{i}, \quad \forall x \in \mathcal{C}_{i}, \quad \forall j \in \mathbb{Z}_{[1,8]}
$$

Next, $\forall x \in \mathcal{C}_{i}, \exists \alpha_{j} \in \mathbb{R}_{[0,1]}$ with $\sum_{j=1}^{8} \alpha_{j} x_{j}=x$ and $\sum_{j=1}^{8} \alpha_{j}=1$. Repeating the above reasoning for Eq. (12) yields Eq. (10), which completes the proof.

For each cube $\mathcal{C}_{i}$, we have to find the output bounds, $y_{i, \min }$ and $y_{i, \max }$, which will be useful for point location. The lower bound $y_{i, \text { min }}$ is calculated as $y_{i, \min }=H_{i} x_{m}+h_{i}$, where $x_{m}$ can be found by solving the following LP:

$$
\min _{x_{m} \in \mathcal{C}_{i}} H_{i} x_{m}+h_{i}
$$

The upper bound $y_{i, \max }$ is calculated as $y_{i, \max }=H_{i} x_{M}+h_{i}$, where $x_{M}$ can be found by solving the following LP:

$$
\min _{x_{M} \in \mathcal{C}_{i}}-\left(H_{i} x_{M}+h_{i}\right)
$$

The partitioning of the state-space can be performed using the adaptive procedure described by Algorithm 1 . 


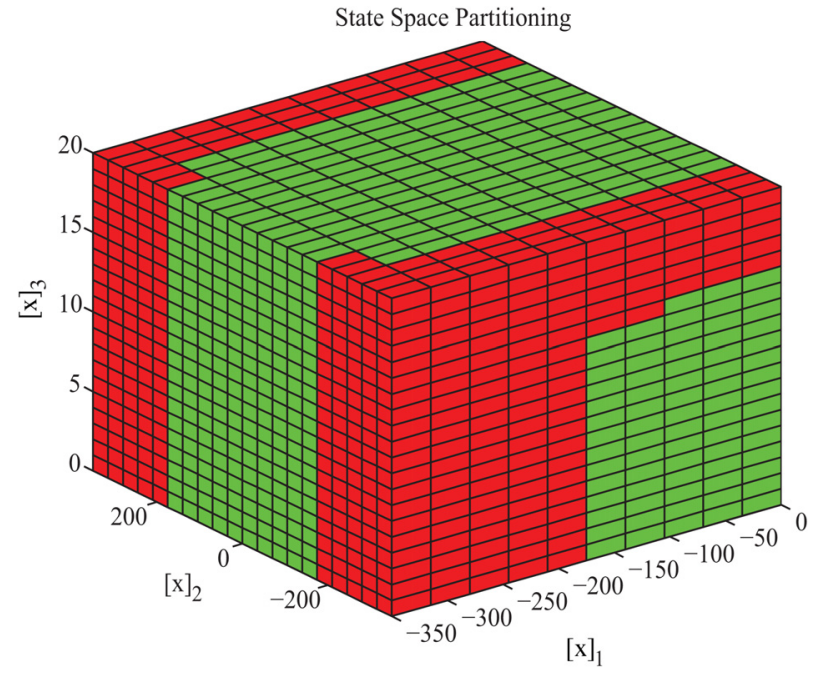

Fig. 2 State-space partitioning in $\mathbf{4 0 0 0}$ cubes

Algorithm 1 Input: Number of gridding points for each state, $\left(k_{1}, k_{2}, k_{3}\right) \in \mathbb{Z}_{\geq 1}^{3}$ and maximum admissible error over all $\mathcal{C}_{i}, \varepsilon_{c} \in \mathbb{R}_{+}$. Output: State-space partition $\left\{\mathcal{C}_{i}\right\}_{i \in \mathbb{Z}_{[1, n]}}$.

1: do state-space partitioning into $n=k_{1} \times k_{2} \times k_{3}$ cubes and solve Eq. (8) for each cube

2: $\quad$ while $\max _{i \in \mathbb{Z}_{[1, n]}} e_{i}>\varepsilon_{c}$ do

3: $\quad$ for $i \leftarrow 1, n$ do

4: $\quad$ if $e_{i}>\varepsilon_{c}$ then

5: $\quad$ split $\mathcal{C}_{i}$ into eight (or four) identical cubes and solve Eq. (8) for each cube

6: $\quad$ end if

7: end for

8: end while

To comply with constraints specified by Eq. ( $4 a)$ and to reduce the complexity all cubes $\mathcal{C}_{i}$ where state or output constraints, described by Eqs. $(4 a)$ and $(4 d)$, are not satisfied are removed. In Fig. 2, the state-space partitioning in 4000 cubes is presented. Cubes which correspond to the case when output or state constraints are not satisfied can be removed.

Next, let us grid the output space into $q \in \mathbb{Z}_{>2}$ equidistantly placed points. The distance between any two consecutive points is defined as $y_{\text {step }}=\left(y_{\max }-y_{\min }\right) /(q-1)$. Now, let us define the grid vector of the output space as

$$
\mathbf{y}:=\left[\begin{array}{lllll}
y_{\min } & \ldots & y_{\min }+p y_{\text {step }} & \ldots & y_{\max }
\end{array}\right]^{\mathrm{T}}, \quad p \in \mathbb{Z}_{[0, q-2]}
$$

In what follows, we seek for the set of indexes that correspond to the cubes that contain the desired value $y_{\text {ref }} \in \mathbf{y}$, i.e.,

$$
I\left(y_{\text {ref }}\right):=\left\{i \in \mathbb{Z}_{[1, n]} \mid y_{\text {ref }} \in \mathcal{C}_{i}\right\}
$$

The output gridding and the cubes indexed by $I\left(y_{\text {ref }}=100\right)$ are illustrated in Fig. 3.

Then, for each $I\left(y_{\text {ref }}\right), y_{\text {ref }} \in \mathbf{y}$, we can generate the explicit state reference, $x_{\text {ref }}^{i}$ by solving a feasibility LP problem, i.e.,

$$
\begin{gathered}
\min _{x_{\mathrm{ref}}^{i}} 0 \\
\text { subject to } \\
H_{i} x_{\mathrm{ref}}^{i}+h_{i}=y_{\mathrm{ref}} \\
x_{\mathrm{ref}}^{i} \in \mathcal{C}_{i}
\end{gathered}
$$

According to Eq. (10), we know that using any $x_{\text {ref }}^{i}$ the output of the system will be in the range $\left[y_{\text {ref }}-\varepsilon_{c}, y_{\text {ref }}+\varepsilon_{c}\right]$, which means

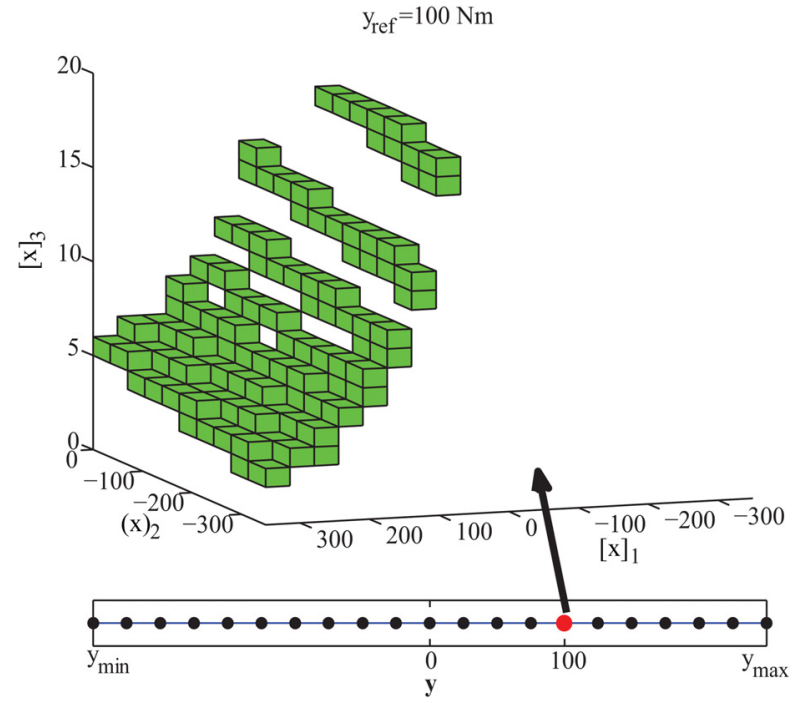

Fig. 3 Output gridding and cubes indexed by $l\left(y_{\text {ref }}=100\right)$ for a state-space partitioning in 4000 cubes

that $x_{\mathrm{ref}}^{i}$ is in a $\varepsilon$-vicinity of a desired solution. This implies that $\varepsilon_{c}$ can be further reduced by considering $x_{\text {ref }}^{i}$ as the initial solution in the original nonlinear problem for each $I\left(y_{\text {ref }}\right), y_{\text {ref }} \in \mathbf{y}$, i.e.,

$$
\begin{gathered}
\min _{x_{\mathrm{ref}}^{i n}} 0 \\
\text { subject to } \\
x_{\mathrm{ref}}^{i \top} C x_{\mathrm{ref}}^{i}-y_{\mathrm{ref}}=0 \\
x_{\mathrm{ref}}^{i} \in \mathcal{C}_{i}
\end{gathered}
$$

Although Eq. (14) is still a nonlinear nonconvex optimization problem, the exploration space $\mathbb{X}$ is reduced to $\mathcal{C}_{i}$, which is a significant improvement compared to Refs. [3] and [8].

Next, let $\mathbf{y} \in \mathbb{Y}^{q}$, with $q \in \mathbb{Z}_{\geq 2}$. Given an arbitrary $y_{\text {ref }} \in \mathbb{Y}$, there exists $p \in \mathbb{Z}_{[0, q-2]}$ such that $\mathbf{y}_{p} \leq y_{\text {ref }}<\mathbf{y}_{p+1}$ and let $e:=\left|\mathbf{y}_{p+1}-\mathbf{y}_{p}\right|, \forall p \in \mathbb{Z}_{[0, q-2]}$. Then, the following inequality holds:

$$
\left|y_{\text {ref }}-\mathbf{y}_{p}\right|<e
$$

Notice that there are multiple state references for each grid point of the output. We propose an online minimization of the power losses to select an energy-optimal state reference out of the set of feasible references, as described in Algorithm 2.

Algorithm 2 Input: The system reference, $y_{\text {ref }} \in \mathbb{Y}$. Output: State reference $x_{\text {ref }}^{*}$.

1: $P_{\text {loss,min }} \leftarrow P_{n}$

2: $\quad$ for $i \leftarrow 1$, length $I\left(y_{\text {ref }}\right)$ do

3: $\quad$ if Online optimization then

4: $\quad$ Find $x_{\text {ref }}^{i}$ by solving Eq. (13)

5: $\quad$ else

6: $\quad$ Use $x_{\text {ref }}^{i}$ obtained by solving offline Eq. (13) (and

$$
\text { Eq. (14)) }
$$

$$
\text { end if }
$$

Evaluate Eqs. (5) and (A1)

if Inequality Eq. (5) is satisfied then

10: $\quad$ if $P_{\text {loss,min }}>P_{\text {loss }}$ then

$$
\text { 11: } \quad P_{\text {loss,min }} \leftarrow P_{\text {loss }}
$$$$
\text { 12: } \quad \text { index } \leftarrow i
$$

13: end if

14: end if

15: end for

16: $x_{\text {ref }}^{*} \leftarrow x_{\text {ref }}^{\text {index }}$. 


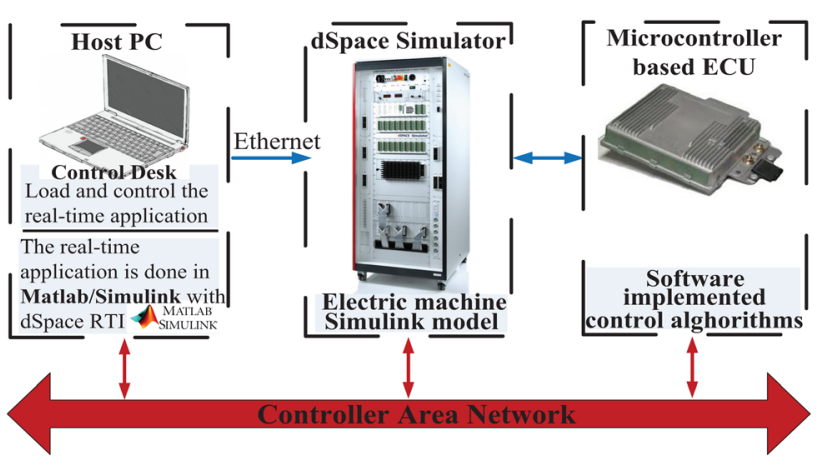

Fig. 4 Real-time test-bench

It should be noted that for reducing the processing time some parts of Eqs. (A1) and (5) can be precalculated offline. The proposed algorithm can be used also for offline state reference generation.

The error bound for the full operational state-space is known as it is stated in the following theorem.

THEOREM 1. Let $\varepsilon_{c} \in \mathbb{R}_{+}$, the error introduced by the PWA approximation of $E q$. ( $3 b$ ) and $e \in \mathbb{R}_{+}$, the error introduced by the output space gridding, be given. Then, for all $y_{\mathrm{ref}} \in \mathbb{Y}$ and corresponding state reference $x_{\mathrm{ref}}^{*}$ obtained via Algorithm 2, the following inequality holds:

$$
\left|y_{\mathrm{ref}}-x_{\mathrm{ref}}^{* \top} C x_{\mathrm{ref}}^{*}\right|<\varepsilon_{c}+e
$$

Proof. Let us start by observing that $\left|y_{\text {ref }}-x_{\text {ref }}^{* \top} C x_{\text {ref }}^{*}\right|=\mid y_{\text {ref }}$ $-\mathbf{y}_{p}+\mathbf{y}_{p}-x_{\text {ref }}^{* \top} C x_{\text {ref }}^{*}|\leq| y_{\text {ref }}-\mathbf{y}_{p}|+| \mathbf{y}_{p}-x_{\text {ref }}^{* \top} C x_{\text {ref }}^{*} \mid$. Next, using Lemma 1 and Eq. (15) yields Eq. (16), which completes the proof.

Note that the inequality Eq. (16) holds true for all $y_{\mathrm{ref}} \in \mathbb{Y}$ and any corresponding $x_{\text {ref }}^{i}$.

\section{Real-Time Results}

4.1 Real-Time Simulation Setup. The HIL test-bench setup for the real-time simulation of the EESM based electrical drive, which is designed to validate the proposed state reference generation algorithm, consists of: Host PC, dSPACE Simulator, microcontroller based ECU, CAN network, power supply, a SIMULINK model for the EESM, and a graphical user interface (GUI) as it can be seen in Fig. 4.

The dSPACE simulator consists of the base board DS5202 and it performs the EESM model calculations. It has an Ethernet interface for direct connection to the host PC which can be used for loading models and reading or adjusting parameters. The host PC runs the GUI, designed in dSPACE ControlDesk to communicate with the real-time dSPACE simulator.

The real-time interface (RTI) is the link between the dSPACE simulator hardware and the MATLAB/SIMULINK model. Real-time workshop (RTW) generates the model code which is compiled and downloaded in the dSpace simulator hardware.

The ECU consists of a 32-bit microcontroller and it performs the operations required to run Algorithm 2. The ECU has similar features with the ECUs used in automotive electrical traction drives, including also a floating point unit. Note that the ECU and the dSPACE simulator exchange data via a CAN bus. Also, the Host PC has access to the CAN bus through the dSPACE ControlDesk application.

4.2 Control Algorithm. The control architecture presented in Fig. 5 is employed in order to demonstrate the efficiency of the proposed algorithm.

Journal of Dynamic Systems, Measurement, and Control

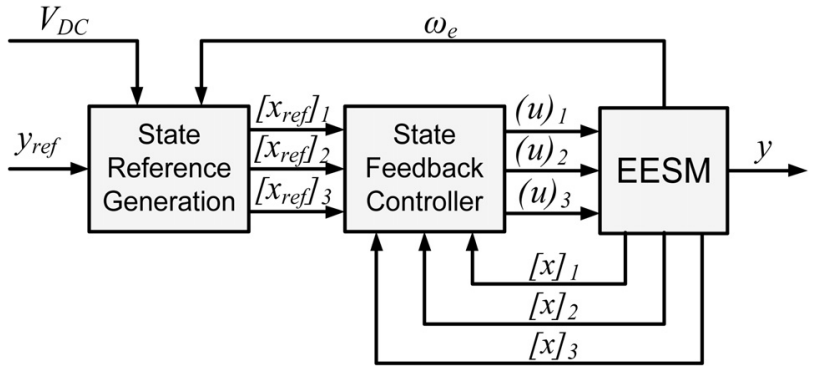

Fig. 5 Control architecture for simulation

A state feedback control law was computed using a LQR controller. For computing the LQR law, the following change of coordinates over inputs was defined:

$$
u=\alpha(x)+\beta(x) \tilde{u}
$$

where, $\alpha(x)=\left[\begin{array}{c}-\omega_{e} L_{q}[x]_{2} \\ \omega_{e}\left[L_{d}[x]_{1}+M_{d}[x]_{3}\right] \\ 0\end{array}\right], \beta(x)=I_{3}$, and $\tilde{u}=[\tilde{u}]_{1}$ $\left.[\tilde{u}]_{2}[\tilde{u}]_{3}\right]^{\top}$.

Using the transformation described by Eq. (17) in Eq. (3a) and discretizing the resulting model, the following discrete-time linear model was obtained:

$$
x(k+1)=A_{d} x(k)+B_{d} \tilde{u}(k)
$$

where $A_{d}=I_{3}+T_{s} A(0)$ and $B_{d}=T_{s} B$. We recall that in Eq. ( $\left.3 a\right)$ the system matrix $A\left(\omega_{e}\right)$ is time variable because it depends of the rotor electrical angular velocity. However, using the transformation described by Eq. (17) results in a linear system without any influence from the rotor electrical angular velocity and, therefore, $A(0):=A\left(\omega_{e}=0\right)$ is used.

In order to achieve offset-free performance, the system state in Eq. (18) was augmented with the integral of the tracking error, denoted by $x_{e}$, with $x_{e}(k+1)=T_{s} x_{e}(k)+\left(x(k)-x_{\text {ref }}(k)\right)$. The augmented system was obtained

$$
z(k+1)=A_{e} z(k)+B_{e} \tilde{u}(k)
$$

where $A_{e}=\left[\begin{array}{cc}A_{d} & O_{3} \\ I_{3} & I_{3}\end{array}\right], B_{e}=\left[\begin{array}{l}B_{d} \\ O_{3}\end{array}\right]$, and $z(k)=\left[\begin{array}{ll}x(k) & x_{e}(k)\end{array}\right]^{\top}$.

Next, a stabilizing controller $K_{\mathrm{opt}}=\left[\begin{array}{ll}K & K_{e}\end{array}\right]$ was designed for the linear model described by Eq. (19) in closed-loop with $\tilde{u}(k)=K x(k)+K_{e}\left(T_{s} x_{e}(k)+x(k)-x_{\text {ref }}(k)\right)$. The cost function

$$
J(z(k), \tilde{u}(k))=\sum_{k=0}^{\infty}\left(z^{\top}(k) Q z(k)+\tilde{u}^{\top}(k) P \tilde{u}(k)\right)
$$

with $Q=\operatorname{Diag}\left(10^{-7}, 10^{-7}, 0.001,0.1,0.001,6\right), \quad P=\operatorname{Diag}$ $\left(2.5 \times 10^{-6}, 2.5 \times 10^{-8}, 3 \times 10^{-8}\right)$ was employed in the corresponding discrete-time infinite horizon optimization problem. It should be noted that there is not a specific rule for choosing the weight matrices. However, a method which can be used as a starting point for a trial-and-error procedure was suggested in Ref. [19]. This method proposes to select $Q$ and $P$ as diagonal matrices with $[Q]_{i i}$ as the inverse of the square of the maximum value of the corresponding state $z_{i}$ and $[P]_{j j}$ as the inverse of the square of the maximum value of the corresponding input $\tilde{u}_{j}, i \in \mathbb{Z}_{[1,6]}$ and $j \in \mathbb{Z}_{[1,3]}$. Therefore, taking into account the values of the existing bounds of the states and inputs results in very small values for the elements of $Q$ and $P$. Nevertheless, these values are within the limits of machine precision and yield the following controller feedback matrices: 


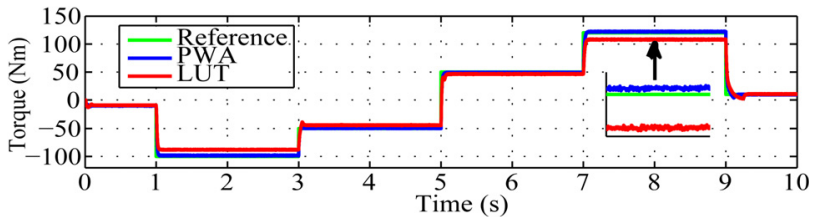

Fig. 6 System output

$$
\begin{array}{r}
K=\left[\begin{array}{ccc}
-0.1343 & 0 & -0.79 \\
0 & -1.3675 & 0 \\
-2.9465 & 0 & -204.0545
\end{array}\right] \\
K_{e}=\left[\begin{array}{ccc}
74.1679 & 0 & 9.0425 \\
0 & 135.2707 & 0 \\
546.09 & 0 & 1.31 \times 10^{4}
\end{array}\right]
\end{array}
$$

The sampling period for the state-feedback control was chosen $T_{s}=100$ microseconds while the sampling period for the state reference generation was chosen 10 milliseconds. This choice is justified by the system dynamics and it is a common choice in the automotive electrical traction drives.

4.3 Measurement Results. The EESM considered in this paper is specific for automotive electrical traction drives. The specifications of the considered EESM are given in Appendix B. The simulation results based on the proposed algorithm are compared with the ones obtained using an offline state reference generation algorithm, based on existing solutions presented in Refs. $[3,8,9]$, and implemented as a look-up table (LUT) for each state.

The simulation scenario presented is realistic because it takes into account also the variation of the machine parameters. The state-space was partitioned into 5000 cubes using $\varepsilon_{c}=12 \mathrm{~N} \mathrm{~m}$ and the output space was gridded into 501 points, which resulted in $e=1 \mathrm{~N} \mathrm{~m}$ in Eq. (15). It should be noted that in each cube the machine parameters were obtained using measurements from a real system. The state reference was obtained by solving offline Eqs. (13) and (14). For implementation of the proposed solution the obtained references are stored in the on-board memory in floating point. This requires $117.2 \mathrm{kB}$ of memory. The set of indexes that correspond to the cubes that contain the desired torque reference is stored in the on-board memory using unsigned type. This yields 2 bytes for each index.

Then, the remaining computations that have to be executed in real-time are the calculation of the set of indexes and the energyoptimal selection of the state reference. Therefore, it can be seen
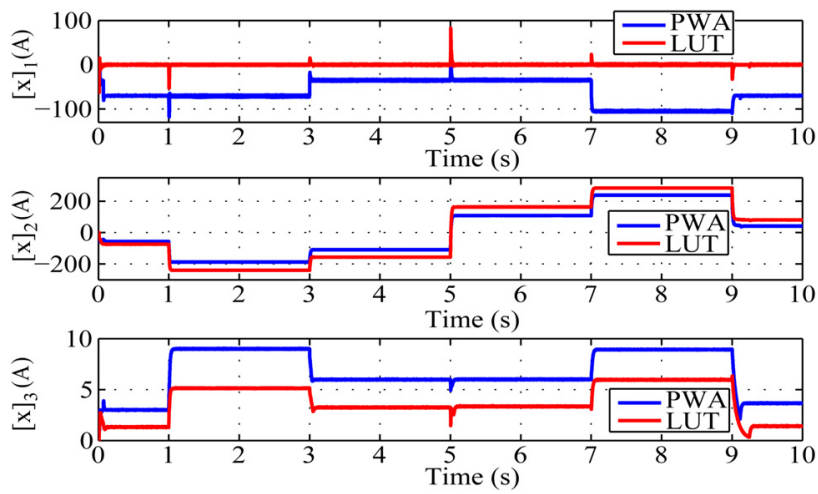

Fig. 7 System states

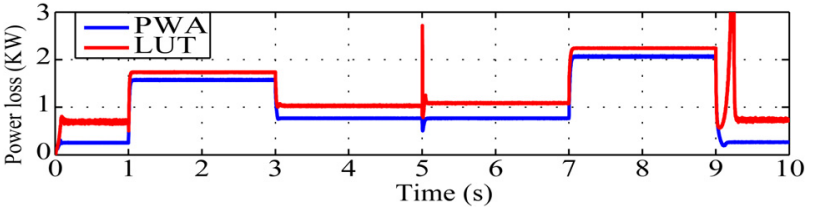

Fig. 8 Estimated power losses

Table 1 Summarized results

\begin{tabular}{lcc}
\hline \hline & Mean output error $(\mathrm{N} \mathrm{m})$ & Mean power losses $(\mathrm{KW})$ \\
\hline PWA & 1.65 & 1.08 \\
LUT & 7.48 & 1.38 \\
\hline \hline
\end{tabular}

that the proposed solution is computational efficient and requires less on-board memory compared to the existing solutions in the automotive industry, which typically involve complex LUTs, see e.g., Ref. [3]. A constant rotor electrical angular velocity, i.e., $\omega_{e}=838 \mathrm{rad} / \mathrm{s}$, and a constant DC link voltage, i.e., $V_{\mathrm{DC}}=300 \mathrm{~V}$ were chosen for the real-time simulation. A $1 \mathrm{~ms}$ sample rate was used for the acquisition of the signals.

The reference and obtained output are plotted in Fig. 6. It can be seen that at high torque the deviation between the reference and measured output increases when the existing solution is employed. The output obtained using the proposed algorithm is much closer to the reference.

Figure 7 shows the comparative evolution of the system states. It can be seen that the state $x_{3}$ is slightly greater and the state $x_{2}$ is slightly lower when the proposed algorithm is employed. The state $x_{1}$ takes negative values when the proposed solution is employed, which results in a better use of the reluctance torque.

Figure 8 shows the evolution of the estimated power losses. It can be seen that the estimated power losses are lower when the proposed algorithm is employed.

Table 1 summarizes the results. It can be seen that the proposed algorithm provides superior performance. The steady-state torque error is approximately four times lower when the proposed algorithm is used. Also it can be seen that the estimated power losses are approximately $20 \%$ lower if the proposed algorithm is employed.

\section{Conclusions}

A new algorithm for the state reference generation with application in EESM torque control was developed based on a proper gridding of the state and output spaces. The proposed algorithm provides high accuracy and robustness to parameter variation (stator inductances and mutual inductance between stator and rotor windings) taking into account also the minimization of the machine power losses. The algorithm is particularly suited for torque control in the high efficiency EESM drives such as automotive propulsion applications.

The proposed algorithm can be used also for offline state reference generation. Then, it can be implemented in a microcontroller using a set of LUTs if the digital memory does not represent a limitation.

The results obtained in real-time illustrate a good behavior of the proposed algorithm when compared with the existing solutions on the aspects of torque error and estimated power losses. It was shown that the steady-state torque error was reduced significantly. Also, it was shown that the estimated power losses are reduced with approximately $20 \%$.

Due to its modular structure, the proposed algorithm can be adapted also to the other types of synchronous machines such as PMSMs or hybrid excited synchronous machines. 


\section{Appendix A: Power Losses Model}

The power losses can be defined as follows:

$$
P_{\text {loss }}(x)=P_{\mathrm{Cu}}(x)+P_{\mathrm{Fe}}(x)+P_{s}(x)
$$

where $P_{\mathrm{Cu}}(x)$ represents the copper losses, $P_{\mathrm{Fe}}(x)$ represents the iron losses, and $P_{s}(x)$ represents the stray losses. The copper losses can be calculated as

$$
P_{\mathrm{Cu}}(x)=\frac{3}{2} R_{s}\left([x]_{1}^{2}+[x]_{2}^{2}\right)+R_{e}[x]_{3}^{2}
$$

The peak value of the flux density can be described as follows:

$$
B_{m}(x)=\sqrt{\left[\left(L_{d}[x]_{1}+M_{d}[x]_{3}\right)^{2}+\left(L_{q}[x]_{2}\right)^{2}\right]\left(\frac{B_{0}}{M_{d}[x]_{3}}\right)^{2}}
$$

where $B_{0}$ is the peak value of the flux density at no load.

Using the peak value of the flux density described by Eq. (A2) and the rotor electrical frequency $f=\omega_{e} / 2 \pi$, the iron losses per unit mass are calculated using the formula presented in Ref. [20]

$$
\frac{P_{\mathrm{Fe}}(x)}{m_{\mathrm{Fe}}}=\left(k_{h} B_{m}(x)^{2} f+k_{e}\left(B_{m}(x) f\right)^{2}+k_{a}\left(B_{m}(x) f\right)^{\frac{3}{2}}\right)
$$

where $k_{h}, k_{e}$, and $k_{a}$ are hysteresis, eddy-current, and excess loss coefficients linked to the magnetic material and $m_{\mathrm{Fe}}$ is the mass of iron. The stray losses can be defined as

$$
P_{s}(x)=4 k_{s} P_{n}\left(\frac{f\left([x]_{1}^{2}+[x]_{2}^{2}\right)}{I_{s, n}^{2} f_{n}}\right)
$$

where $P_{n}, I_{s, n}$, and $f_{n}$ are the nominal values of power, stator phase current, and electrical frequency, respectively, and $k_{s}$ is the stray constant.

\section{Appendix B: EESM Parameters}

\section{Parameter}

Nominal power $\left(P_{n}\right)$

Maximum Torque $\left(T_{\max }\right)$

Nominal DC link voltage $\left(V_{\mathrm{DC}, n}\right)$

Nominal phase current $\left(I_{s, n}\right)$

Maximum phase current $\left(I_{s, \max }\right)$

Minimum stator and excitation

currents $\left(x_{\min }\right)$

Maximum stator and excitation

currents $\left(x_{\max }\right)$

Nominal electrical angular

velocity $\left(\omega_{e, n}\right)$

Maximum electrical angular

velocity $\left(\omega_{e, \max }\right)$

Nominal electrical frequency $\left(f_{n}\right)$

Number of pole pairs $\left(P_{p}\right)$

Stator resistance at $20^{\circ} \mathrm{C}\left(R_{s}\right)$

Excitation resistance at $20^{\circ} \mathrm{C}\left(R_{e}\right)$ $d$-axis inductance $\left(L_{d}\right)$

$1.488 \mu \mathrm{H}$

$q$-axis inductance $\left(L_{q}\right)$

Mutual inductance $\left(M_{d}\right)$

$2.264 \mu \mathrm{H}$

Excitation inductance $\left(L_{e}\right)$

$90.6 \mu \mathrm{H}$

Peak value of the flux density at

no load $\left(B_{0}\right)$

Hysteresis loss coefficient $\left(k_{h}\right)$

Eddy-current loss coefficient $\left(k_{e}\right)$

Excess loss coefficient $\left(k_{a}\right)$

Mass of iron $\left(m_{\mathrm{Fe}}\right)$

Stray constant $\left(k_{s}\right)$

$0.6 \mathrm{H}$

$1.5 \mathrm{~T}$

$7.10 \times 10^{-3} \mathrm{~J} / \mathrm{kg} \cdot \mathrm{T}^{2}$

$2.33 \times 10^{-4} \mathrm{~J} \cdot \mathrm{s} / \mathrm{kg} \cdot \mathrm{T}^{2}$

$3.72 \times 10^{-4} \mathrm{~J} \cdot \mathrm{s}^{1 / 2} / \mathrm{kg} \cdot \mathrm{T}^{3 / 2}$

$16.7 \mathrm{~kg}$

0.0025

\section{References}

[1] de Santiago, J., Bernhoff, H., Ekergård, B., Eriksson, S., Ferhatovic, S., Waters, S., and Leijon, M., 2012, "Electrical Motor Drivelines in Commercial All-Electric Vehicles: A Review,” IEEE Trans. Veh. Technol., 61(2), pp. 475-484.

[2] Hagstedt, D., Marquez, F., and Alakula, M., 2008, "A Comparison Between PMSM, EMSM and SMSM in a BAS Application," International Conference on Electrical Machines, Vilamoura, Sept. 6-9, pp. 1-5.

[3] Märgner, M., and Hackmann, W., 2010, "Control Challenges of an Externally Excited Synchronous Machine in an Automotive Traction Drive Application," Emobility-Electrical Power Train, pp. 1-6.

[4] Park, R. H., 1929, "Two-Reaction Theory of Synchronous Machines. Generalized Method of Analysis-Part I," AIEE Trans., 48(3), pp. 716-727.

[5] Krause, P. C., Wasynczuk, O., Sudhoff, S. D., and Pekarek, S., 2013, Analysis of Electric Machinery and Drive Systems, 3rd ed., Wiley-IEEE Press, Hoboken, NJ.

[6] Chai, S., Wang, L., and Rogers, E., 2013, "A Cascade MPC Control Structure for a PMSM With Speed Ripple Minimization,” IEEE Trans. Ind. Electron., 60(8), pp. 2978-2987.

[7] Jain, A. K., and Ranganathan, V. T., 2011, "Modeling and Field Oriented Control of Salient Pole Wound Field Synchronous Machine in Stator Flux Coordinates,” IEEE Trans. Ind. Electron., 58(3), pp. 960-970.

[8] Girardin, A., and Friedrich, G., 2006, "Optimal Control for a Wound Rotor Synchronous Starter Generator," IEEE Industry Applications Conference, Tampa, FL, Oct. 8-12, Vol. 1, pp. 14-19.

[9] Janiaud, N., Vallet, F., Petit, M., and Sandou, G., 2010, "Electric Vehicle Powertrain Simulation to Optimize Battery and Vehicle Performances," IEEE Vehicle Power and Propulsion Conference, Lille, France, Sept. 1-3, pp. 1-5.

[10] de Kock, H. W., Rix, A. J., and Kamper, M. J., 2010, “Optimal Torque Control of Synchronous Machines Based on Finite-Element Analysis," IEEE Trans. Ind. Electron., 57(1), pp. 413-419.

[11] Carpiuc, S. C., Patrascu, D. I., and Lazar, C., 2011, "Optimal Torque Control of the Interior Permanent Magnet Synchronous Machine," 23rd International Symposium on Information, Communication and Automation Technologies, Sarajevo, Bosnia, Oct. 27-29, pp. 1-8.

[12] Carpiuc, S. C., Lazar, C., and Patrascu, D. I., 2012, "Optimal Torque Control of the Externally Excited Synchronous Machine,” J. Control Eng. Appl. Inform., 4(2), pp. 80-88.

[13] Ulsoy, A. G., Peng, H., and Cakmakci, M., 2012, Automotive Control Systems, Cambridge University Press, New York.

[14] Salehi, R., Shahbakhti, M., and Hedrick, J. K., 2014, "Real-Time Hybrid Switching Control of Automotive Cold Start Hydrocarbon Emission,” ASME J. Dyn. Syst., Meas., Control, 136(4), p. 041002.

[15] Monot, A., Navet, N., Bavoux, B., and Simonot-Lion, F., 2012, "Multisource Software on Multicore Automotive ECUs-Combining Runnable Sequencing With Task Scheduling," IEEE Trans. Ind. Electron., 59(10), pp. 3934-3942.

[16] Carpiuc, S., and Lazar, M., 2012, "Efficient State Reference Generation for Torque Control in Externally Excited Synchronous Machines," 16th International Conference on System Theory, Control and Computing, pp. 1-7.

[17] Retif, J. M., Lin-Shi, X., Llor, A. M., and Morand, F., 2004, "New Hybrid Direct-Torque Control for a Winding Rotor Synchronous Machine," 35th Annual IEEE Power Electronics Specialists Conference, Aachen, Germany, June 20-25, Vol. 2, pp. 1438-1442.

[18] Lazar, M., 2010, "On Infinity Norms as Lyapunov Functions: Alternative Necessary and Sufficient Conditions," 49th IEEE Conference on Decision and Control, Atlanta, GA, Dec. 15-17, pp. 5936-5942.

[19] Bryson, A. E., and Ho, Y. C., 1975, Applied Optimal Control: Optimization, Estimation, and Control. Taylor and Francis Group, New York.

[20] Bertotti, G., 1988, "General Properties of Power Losses in Soft Ferromagnetic Materials,” IEEE Trans. Magn., 24(1), pp. 621-630. 